\title{
Maternal sevoflurane exposure affects differentiation of hippocampal neural stem cells by regulating miR-410-3p and ATN1
}

\author{
Yi Zhang, Ziyi Wu, Xingyue Li, Yuxiao Wan, Yinong Zhang and Ping Zhao* (D)
}

\begin{abstract}
Background: Currently, numerous animal studies have shown that exposure to commonly used general anesthetics during pregnancy may cause neurocognitive impairment in the offspring. Reportedly, exposure to sevoflurane during mid-trimester of pregnancy can inhibit proliferation of neural stem cells (NSCs) and lead to early apoptosis. Whether exposure to sevoflurane during pregnancy affects the differentiation of NSCs remains unclear.

Methods: In the present study, pregnant rats were exposed to $3 \%$ sevoflurane once for $2 \mathrm{~h}$ on gestational day 14 (G14) or 3 times for $2 \mathrm{~h}$ on G13, G14, and G15. Next, the differentiation of NSCs was measured using neuron marker $\beta$-tubulin III and astrocyte marker glial fibrillary acidic protein (GFAP) in fetal brain tissues $24 \mathrm{~h}$ and $72 \mathrm{~h}$ after anesthesia and in hippocampus on postnatal day 28. Primary cultured rat NSCs were exposed to $4.1 \%$ sevoflurane to explore the mechanism.
\end{abstract}

Results: The results showed that during mid-trimester, multiple exposures to sevoflurane can cause premature differentiation of NSCs in developing brains of offspring and lead to long-term neuron reduction and astrocyte proliferation in hippocampus. The data from the present study indicated that repeated exposure to sevoflurane downregulated atrophin-1 (ATN1) expression and caused early differentiation of NSCs. Overexpression of ATN1 via lentivirus transfection attenuated the influence of sevoflurane. Using dual luciferase assay, ATN1 was found to be a target gene of microRNA-410-3p (miR-410-3p). MiR-410-3p suppression via lentivirus transfection recovered the ATN1 expression and differentiation of NSCS.

Conclusions: The results from the present study demonstrated that repeated exposure to sevoflurane leads to early differentiation of NSCs and long-term effects via the miR-410-3p/ATN1 pathway.

Keywords: Sevoflurane, Mid-trimester, Neural stem cells, Differentiation, Neurotoxicity, microRNA

\section{Background}

Numerous animal studies have shown that exposure to anesthesia drugs can lead to long-term neurocognitive impairment in the developing brain. Most research has focused on the newborn period [1-3]. Because the surgical technologies are constantly advancing, the number of non-obstetric surgeries is continually increasing. Mid\footnotetext{
University, Shenyang, China

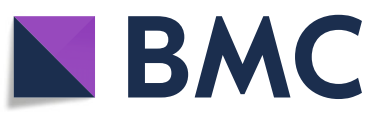

*Correspondence: zhaop@sj-hospital.org; zhaoping_sj@163.com Department of Anesthesiology, Shengjing Hospital of China Medical trimester is considered a relatively safe period to perform surgery during pregnancy. However, the midtrimester is a critical period for fetal brains because neural stem cells (NSCs) undergo high proliferation and differentiation $[4,5]$. In our previous studies [6-8], exposure to sevoflurane in the mid-trimester of pregnancy suppressed NSC proliferation and led to early apoptosis. However, whether sevoflurane affects NSC differentiation remains unclear.

(c) The Author(s). 2020 Open Access This article is licensed under a Creative Commons Attribution 4.0 International License, which permits use, sharing, adaptation, distribution and reproduction in any medium or format, as long as you give appropriate credit to the original author(s) and the source, provide a link to the Creative Commons licence, and indicate if changes were made. The images or other third party material in this article are included in the article's Creative Commons licence, unless indicated otherwise in a credit line to the material. If material is not included in the article's Creative Commons licence and your intended use is not permitted by statutory regulation or exceeds the permitted use, you will need to obtain permission directly from the copyright holder. To view a copy of this licence, visit http://creativecommons.org/licenses/by/4.0/ The Creative Commons Public Domain Dedication waiver (http://creativecommons.org/publicdomain/zero/1.0/) applies to the data made available in this article, unless otherwise stated in a credit line to the data. 
In the present study, the results showed that after sevoflurane exposure, the atrophin-1 (ATN1) level was significantly decreased. ATN1 is highly expressed in brain tissues and mutations in the ATN1 gene can cause a rare neurodegenerative disease, dentatorubralpallidoluysian atrophy (DRPLA) [9]. In most of the previous ATN1 expression studies [10, 11], the focus was on DRPLA. Recently, ATN1 was reported to play an important role in NSC maintenance [12], which is in agreement with our results. Thus, in the present study, whether sevoflurane could affect NSC differentiation by changing the expression of ATN1 was investigated.

In numerous studies, microRNAs (miRNAs) have been reported to play significant roles in neural development $[13,14]$. Several miRNAs were confirmed to participate in NSC apoptosis and differentiation [15, 16]. Abnormal miRNA expression after exposure to anesthesia drugs could cause significant neurocognitive impairments in developing brains $[17,18]$. In the present study, several databases were used to determine if ATN1 is a direct target of miRNA-410-3p (miR-410-3p), which was confirmed using dual-luciferase reporter assay. However, whether miR-410-3p plays a role in NSC differentiation after exposure to sevoflurane remains unknown.

To investigate whether sevoflurane affects NSC differentiation during the early stage of brain development, rat models in mid-trimester of pregnancy and primary cultured NSCs were repeatedly exposed to sevoflurane. We hypothesized that repeated sevoflurane exposure would lead to early NSC differentiation and long-term neuron reduction by regulating the miR-410-3p and ATN1 expression.

\section{Methods}

\section{Animals}

Adult Sprague-Dawley rats were housed in a room with constant temperature of $24 \pm 1{ }^{\circ} \mathrm{C}$ under a 12-h light/12$\mathrm{h}$ dark cycle, with free access to water and food. All experimental procedures were approved by the Institutional Animal Care and Use Committee of Shengjing Hospital, China Medical University (No.2016PS028K), and were conducted following the National Institute of Health Guideline for the Care and Use of Laboratory Animals.

\section{Cell culture and differentiation}

Rat NSCs were isolated from the hippocampus of fetal Sprague-Dawley rats on G14 or G15. The isolated cells were plated in culture flasks at a density of $1 \times 10^{5} / \mathrm{mL}$ and maintained in an atmosphere of $5 \% \mathrm{CO}_{2}$ and $95 \%$ air. The cells were cultured in serum-free medium of Dulbecco's modified Eagle's medium (DMEM)/F12, HEPES (11330032; Gibco, USA) supplemented with $2 \%$ B27 without vitamin A (12587010; Gibco), $20 \mathrm{ng} / \mathrm{mL}$ basic fibroblast growth factor (rat bFGF, 3339-FB-025; R\&D, USA), $20 \mathrm{ng} / \mathrm{mL}$ epidermal growth factor (rat EGF, 3214-EG-100; R\&D), and 1\% penicillinstreptomycin (1512022; Gibco). Half of the culture medium was replaced every other day, and the cells were digested with Accutase (A6964; Sigma-Aldrich, USA). All experiments were performed on cells from passages $2-4$ to reduce experimental deviations. To induce NSC differentiation, rat NSCs were digested into single cells and plated onto plates pre-coated with $0.25 \%$ poly-Dlysine (P6409; Sigma-Aldrich) using differentiation medium of DMEM/F12 supplemented with $2 \%$ B27 without vitamin A, $1 \%$ fetal bovine serum (FBS, 10100139; Gibco), and 1\% penicillin-streptomycin.

HEK293T cells were purchased from Cell Bank of Chinese Academy of Sciences (Shanghai, China) and cultured in high-glucose DMEM (11965084; Gibco) supplemented with 10\% FBS (10100139; Gibco) and antibiotics (1512022; Gibco). Cells were incubated in an atmosphere at $37^{\circ} \mathrm{C}$ in $5 \% \mathrm{CO}_{2}$ and the culture medium replaced every other day.

\section{Sevoflurane exposure}

Two or three adult female Sprague-Dawley rats were caged with one male rat to allow free mating. If sperm or vaginal emboli were detected on the second day, the female rat was marked as G0. For the in vivo experiments, pregnant rats were placed into a $30 \%$ oxygen plastic chamber and exposed to $3 \%$ sevoflurane (1.5 MAC) for $2 \mathrm{~h}$ on G14 (SEV $\times 1$ group; $n=9 /$ group) or on G13, G14, and G15 (SEV $\times 3$ group; $n=9 /$ group). CON group ( $n=9 /$ group) consisted of rats placed in the same conditions without exposure to sevoflurane. The fetal brain tissues were obtained after cesarean section at $24 \mathrm{~h}, 72 \mathrm{~h}$, and postnatal day 28 . For the in vitro experiments, hippocampal NSCs were obtained as described above. The NSCs were inoculated into 24-well plates and randomly allocated into 8 groups: CON group, $4.1 \%$ $\mathrm{SEV} \times 1$ group $(\mathrm{SEV} \times 1), 4.1 \% \mathrm{SEV} \times 3$ group $(\mathrm{SEV} \times 3)$, negative lentivirus group (NC), ATN1-overexpression lentivirus group (LV-ATN1), $4.1 \% \mathrm{SEV} \times 3$ plus ATN1overexpression lentivirus group (LV-ATN $1+\mathrm{SEV} \times 3$ ), miR-410-3p-suppression lentivirus group (LV-410), and 4.1\% $\mathrm{SEV} \times 3$ plus miR-410-3p-suppression lentivirus group $(\mathrm{LV}-410+\mathrm{SEV} \times 3)$. The plates were placed into an incubator containing a mixture of $5 \% \mathrm{CO}_{2}$ and $95 \%$ air, and a gas monitor (Drager, Germany) was used to detect sevoflurane concentration. The $\mathrm{SEV} \times 1$ group was exposed to $4.1 \%$ sevoflurane for $2 \mathrm{~h}$, and all SEV $\times 3$ groups were exposed to $4.1 \%$ sevoflurane for $2 \mathrm{~h}$ on 3 consecutive days. All groups that were not exposed to sevoflurane were kept under the same conditions of a mixture of $5 \% \mathrm{CO}_{2}$ and $95 \%$ air for the same amount of time. 


\section{Western blot}

The brain tissues and collected cells were stored at $80^{\circ} \mathrm{C}$ before use and homogenized to determine protein expression using the western blotting protocol described in our previous study [19]. The protein concentration was measured using the BCA Protein Assay Kit (P0010; Beyotime, China). Electrophoresis was performed using $10 \%$ or $12.5 \%$ SDS-polyacrylamide gels, and then proteins were electrotransferred to polyvinylidene fluoride membranes (IPVH0010; Millipore, Germany). The membranes were blocked with BSA or $5 \%$ non-fat milk and incubated with primary antibodies $\beta$-tubulin III $(1: 1000$, T2200; Sigma-Aldrich), GFAP (1:10,000, ab53554; Abcam, UK), nestin (1:1000, ab6142; Abcam), ATN1 (1: 500, orb213859; Biorbyt, UK), and glyceraldehyde-3phosphate dehydrogenase (GAPDH) (1:1000, 60004-1-Ig; Proteintech, USA) overnight at $4{ }^{\circ} \mathrm{C}$. The membranes were incubated with second antibodies the next day for $2 \mathrm{~h}$ at room temperature, and then photographed using a GE Amersham Imager 600. Number of tissues was 5 per group and number of cells was 3 per group in western blot experiments. Images were analyzed using Image-Pro Plus 6.0 software.

\section{RT-qPCR}

The mRNA in each group was extracted using RNAiso Plus (9108; TaKaRa, China) and miRNA was extracted using RNAiso for small RNAs (9753A; TaKaRa, China). The primers were designed and synthesized by Sangon Biotech (China; Table 1). Reverse transcription and reaction conditions were performed according to the instructions in the PrimeScript ${ }^{\mathrm{mm}}$ RT reagent Kit with gDNA Eraser (RR047A; TaKaRa) and Mir-X miRNA First-Strand Synthesis Kit (638,315; Clontech, USA) using SYBR green (RR420A; TaKaRa, China). Quantitative analysis of gene expression was calculated using $2^{-\Delta \Delta C t}$ method with GAPDH (B661204; Sangon biotech, China) and U6 as internal references.

\section{Immunofluorescence staining}

Brains were immersed in precooled $4 \%$ paraformaldehyde (PFA) for $24 \mathrm{~h}$ followed by dehydration in an ethanol gradient, and then paraffin embedded. Next, the tissues were sliced into sections approximately $3.0-\mu \mathrm{m}$ thick. The sections were deparaffinized and heated in citrate buffer for $7.5 \mathrm{~min}$ at $121{ }^{\circ} \mathrm{C}$. The cells were plated onto glass coverslips pre-coated with poly-D-lysine, and

Table 1 Primer sequences for RT-qPCR

\begin{tabular}{ll}
\hline Gene & Sequence \\
\hline ATN1 & Forward: GTCTTCGTCTCAAGCCGCCTATTC \\
& Reverse: AGGAGGTGGTGATTGGAGGAACTG \\
miR-410-3p & cgcgAATATAACACAGATGGCCTGT \\
\hline
\end{tabular}

then fixed in 4\% PFA for $30 \mathrm{~min}$ at room temperature. To reduce background staining and permeabilize membranes, $10 \% \mathrm{FBS}$ and $0.5 \%$ Triton X-100 were added to the sections and coverslips for $40 \mathrm{~min}$ at room temperature, and then incubated with primary antibodies overnight at $4{ }^{\circ} \mathrm{C}$. The next day, the sections were incubated with secondary antibodies for $2 \mathrm{~h}$ and DAPI for $5 \mathrm{~min}$ at room temperature. The primary antibodies were the following: nestin (1:250, ab92391; Abcam; 1: 300, 4760; Cell Signaling Technology, USA), sexdetermining region Y box 2 (SOX2) (1:500, abs13121950ug; absin), $\beta$-tubulin III (1:250, T2200; SigmaAldrich), GFAP (1:500, ab53554; Abcam), and ATN1 (1: 500 HPA031619; Sigma-Aldrich). The nuclei were stained with DAPI. Sections and coverslips were imaged with Nikon $\mathrm{C} 1$ microscope by an investigator who was blinded to the experimental interventions. The $\beta$-tubulin III and GFAP immunoreactivity was quantified by the ratio of the number of $\beta$-tubulin III or GFAP-positive cells to the total number of cells. Number of rats per group was $5(n=5)$ and number of cell coverslips per group was $3(n=3)$.

\section{Lentivirus transfection}

ATN1 overexpression lentivirus and miR-410-3p suppression lentivirus were purchased from GeneChem Corporation, China. NSCs in 96-well plates were transfected to detect the optimum multiplicity of infection (MOI) and experiments performed in 24-well plates. Medium containing lentivirus was replaced $24 \mathrm{~h}$ posttransfection with fresh medium. Transfection efficiency was confirmed using RT-qPCR.

\section{Dual-luciferase reporter assay}

Dual-luciferase reporter assay was performed in $293 \mathrm{~T}$ cells using the Dual-luciferase Reporter System (Promega, China). All pmirGLO vectors were purchased from GenePharma, China. The pmirGLO-ATN1-WT or pmirGLOATN1-MUT vector was transfected with miR-410-3p mimics or mimics negative control (mimics-NC) into 293 $\mathrm{T}$ cells using Lipo2000 (Invitrogen, USA). Luciferase activity was detected at $24 \mathrm{~h}$ after transfection. The experiments were performed three times independently.

\section{Statistical analysis}

All data were analyzed using GraphPad Prism 7.0 software or SPSS 17.0 for Windows. Results were presented as means \pm standard deviation (SD). Student's $t$ test and one-way analysis of variance (ANOVA) followed by Tukey's post hoc multiple comparison test was used for data analyses. A $p$ value $<0.05$ was considered statistically significant. 


\section{Results}

Confirmation and differentiation of NSCs

To investigate the mechanism in vitro, primary cultured hippocampal NSCs were used. The NSCs were isolated from fetal hippocampi of Sprague-Dawley rats on embryonic (E) day 14 to E15. Observation under microscope showed that NSCs grew in a typical round shape (Fig. 1a), which was confirmed using immunofluorescence with the NSC marker nestin and Sex-determining region Y box 2 (SOX2)(Fig. 1b). For NSC differentiation, NSCs were induced to differentiate for $120 \mathrm{~h}$ which was confirmed using immunofluorescence with neuron marker $\beta$-tubulin III (Fig. 1c) and astrocyte marker glial fibrillary acidic protein (GFAP; Fig. 1d).

\section{Repeated exposure to sevoflurane led to early} differentiation of hippocampal NSCs

To determine whether sevoflurane can affect NSC differentiation, the expression of NSC marker nestin, neuron marker $\beta$-tubulin III, and astrocyte marker GFAP was examined in fetal brains and primary cultured hippocampal NSCs using western blotting and immunofluorescence at $24 \mathrm{~h}$ and $72 \mathrm{~h}$ after single or repeated exposure to sevoflurane (Figs. 2 and 3). After repeated maternal exposure to $3 \%$ sevoflurane, the $\beta$ tubulin III (Fig. 2a, b, d, g) and GFAP levels (Fig. 2a, $\mathrm{c}, \mathrm{e}, \mathrm{h}$ ) were increased and the nestin level (Fig. 2a-c, f) was decreased in fetal brain tissue. However, significant difference was not observed between control $(\mathrm{CON})$ group and single $3 \%$ sevoflurane exposure $(\mathrm{SEV} \times 1)$ group(Fig. $2 \mathrm{a}-\mathrm{h})$. Primary cultured NSCs exposed to $4.1 \%$ sevoflurane once or 3 times $(\mathrm{SEV} \times$
3) showed $\beta$-tubulin III (Fig. 3a, b, d, g), GFAP (Fig. 3a, c, e, h), and nestin (Fig. 3a, c, f) levels corresponded to in vitro results. The results showed that repeated exposure to sevoflurane led to early differentiation in hippocampal NSCs.

\section{Repeated exposure to sevoflurane led to long-term neuron reduction and astrocyte proliferation in hippocampus}

Our previous study showed that a single maternal 3\% sevoflurane exposure does not cause long-term neurocognitive impairment in fetal rats, while repeated exposure of $3 \%$ sevoflurane can cause learning and memory impairment in the offspring [19]. The $\beta$ tubulin III and GFAP levels in rat brains were examined on postnatal day 28 (Fig. 4) as well as in the cultured NSCs (Fig. 5). After repeated exposure to sevoflurane, $\beta$-tubulin III protein was reduced in both postnatal fetal hippocampus (Fig. 4a, b) and cultured NSCs (Fig. 5a, b) compared with the CON group and $\mathrm{SEV} \times 1$ group. GFAP expression was upregulated in postnatal fetal hippocampus (Fig. 4a, c) and cultured NSCs (Fig. 5a, c). Immunofluorescence showed similar results regarding the quantity of neurons and astrocytes in CA1 hippocampal region (Fig. 4d-g). Significant difference was not observed between $\mathrm{CON}$ group and SEV $\times 1$ group.

\section{Exposure to sevoflurane affected NSC differentiation by downregulating ATN1 expression}

The expression of ATN1 was downregulated in both fetal brain tissues (Fig. S1A, B) and primary cultured NSCs (Fig. 6a, e) after repeated exposure to

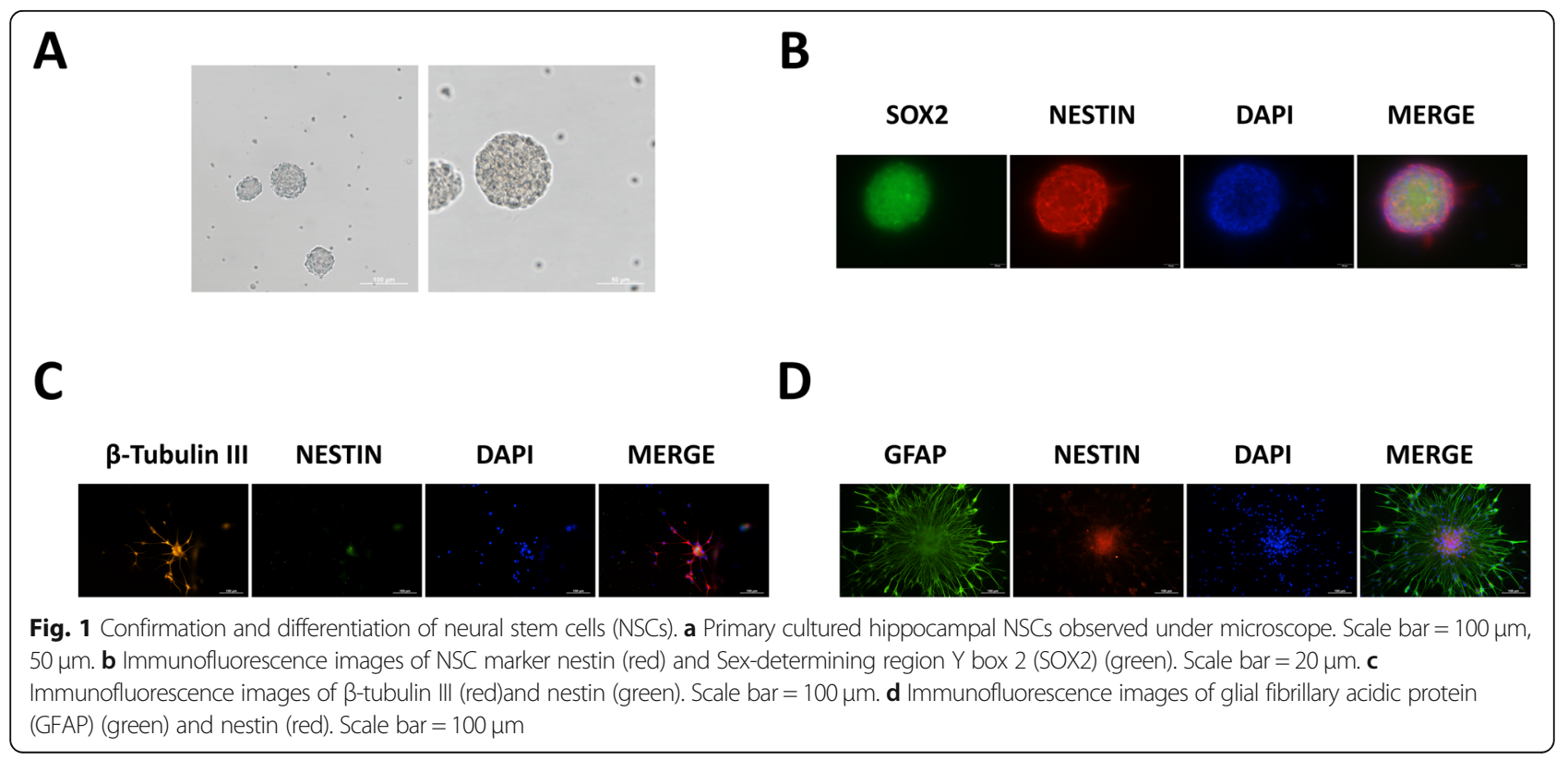



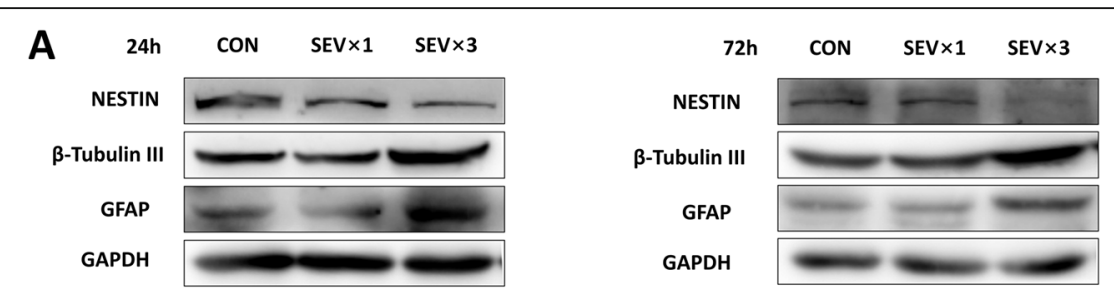

\section{B}
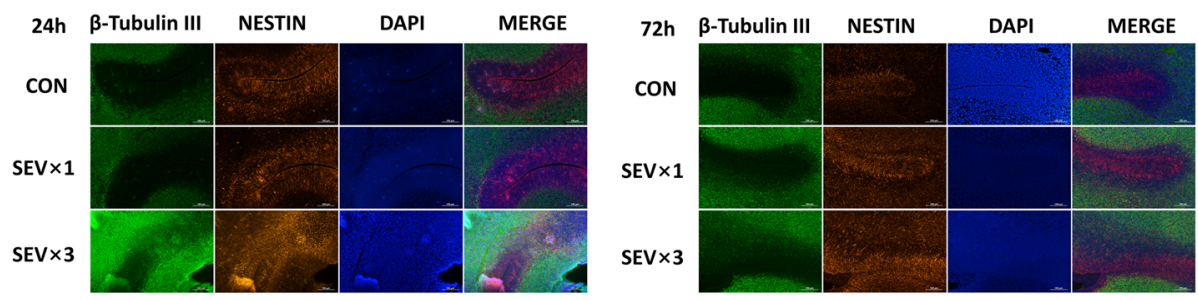

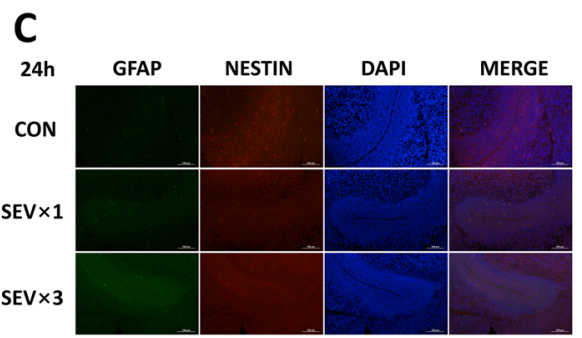

D

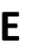

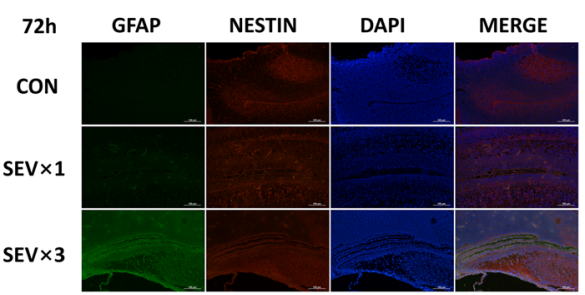

F

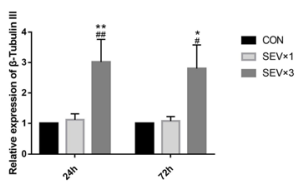

G
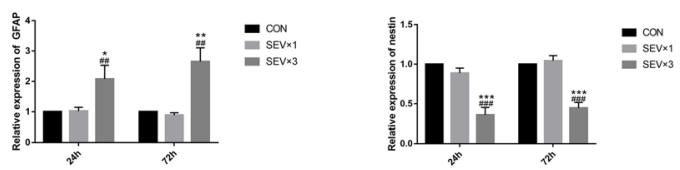

H

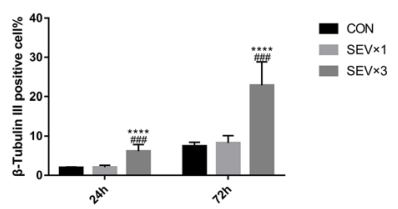

Fig. 2 Repeated maternal exposure to 3\% sevoflurane led to early NSC differentiation in fetal brains. a Western blotting images of $\beta$-tubulin III, GFAP, and nestin. b Immunofluorescence images of $\beta$-tubulin III (green) and nestin (red). Scale bar $=100 \mu$ m. $\mathbf{c}$ Immunofluorescence images of GFAP (green) and nestin (red). Scale bar $=100 \mu \mathrm{m}$. d Quantitative analysis of $\beta$-tubulin III. e Quantitative analysis of GFAP. $\mathbf{f}$ Quantitative analysis of nestin. g Quantification of $\beta$-tubulin III-positive cells. $\mathbf{h}$ Quantification of GFAP-positive cells. Values are means \pm SD $(n=5 / g r o u p)$. ${ }^{*} p<0.05$, ${ }^{* *} p<$ 0.01 , ${ }^{* * *} p<0.001$ compared with control (CON) group; \# $p<0.05$, \#\# $p<0.01$, \#\#\# $p<0.001$ compared with sevoflurane (SEV) $\times 1$ group. One-way analysis of variance (ANOVA) followed by Tukey's post hoc multiple comparison test was used for data analysis

sevoflurane. After transfecting ATN1 overexpression lentivirus into NSCs and exposing to sevoflurane, in the $4.1 \% \mathrm{SEV} \times 3+\mathrm{ATN1}$-overexpression lentivirus $(\mathrm{LV}-\mathrm{ATN} 1+\mathrm{SEV} \times 3)$ group, the levels of $\beta$-tubulin III (Fig. 6a, c, f, k), GFAP (Fig. 6a, d, g, l), and nestin (Fig. 6a, h) were not significantly different than in the CON group at $24 \mathrm{~h}$ and $72 \mathrm{~h}$. In the LV-ATN1+ $\mathrm{SEV} \times 3$ group, $\beta$-tubulin III (Fig. 6a, c, f, k) and GFAP (Fig. 6a, d, g, l) expressions were significantly reduced and nestin (Fig. 6a, h) expression was upregulated compared with the $\mathrm{SEV} \times 3$ group. ATN1 mRNA expression level was detected using reverse transcription quantitative polymerase chain reaction (RT-qPCR; Fig. 6b). $\beta$-tubulin III (Fig. 6a, i) and GFAP (Fig. 6a, j) protein levels were rescued in the $\mathrm{LV}-\mathrm{ATN} 1+\mathrm{SEV} \times 3$ group at day 28 . Results confirmed that sevoflurane affected differentiation of hippocampal NSCs by downregulating ATN1 expression. 

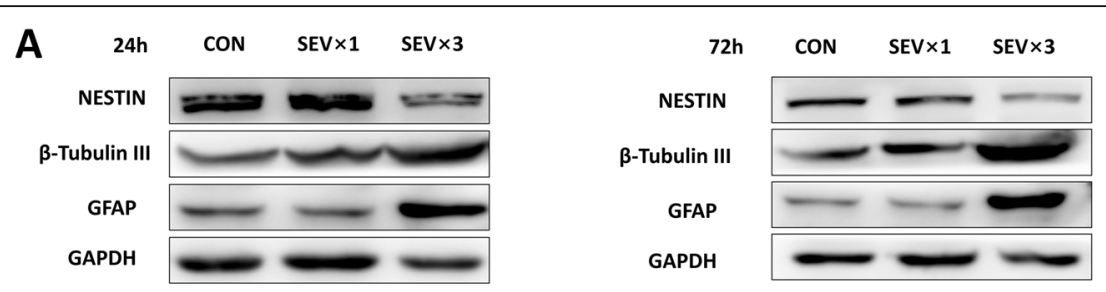

\section{B}
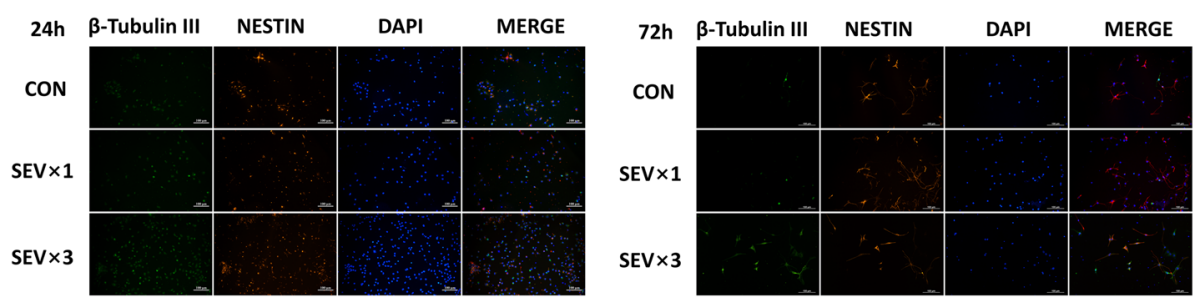

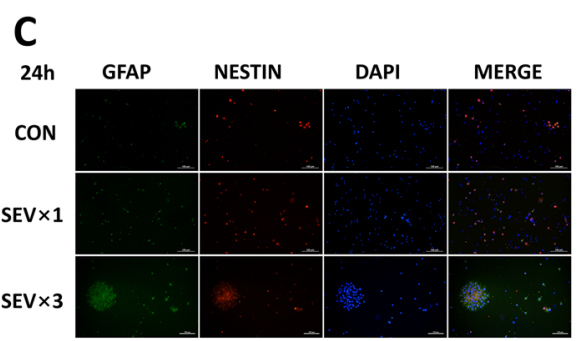

D

E

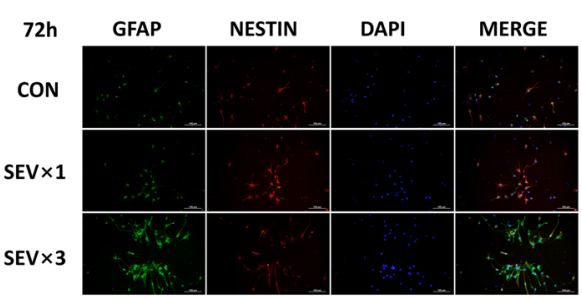

F

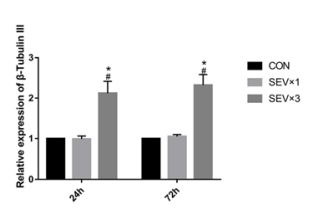

G

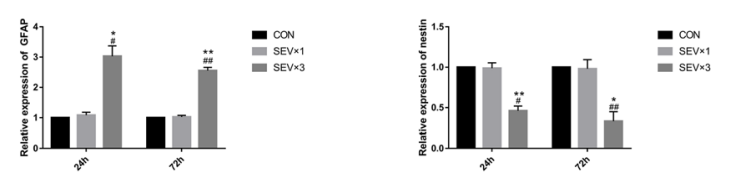

H
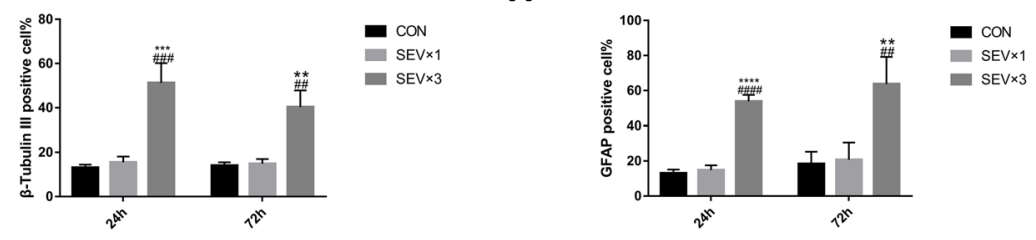

Fig. 3 Repeated exposure to $4.1 \%$ sevoflurane led to early differentiation in primary cultured hippocampal NSCs. a Western blotting images of $\beta$ tubulin III, GFAP, and nestin. b Immunofluorescence images of $\beta$-tubulin III (green) and nestin (red). Scale bar = $100 \mu \mathrm{m}$. $\mathbf{c}$ Immunofluorescence images of GFAP (green) and nestin (red). Scale bar $=100 \mu \mathrm{m}$. d Quantitative analysis of $\beta$-tubulin III. e Quantitative analysis of GFAP. (F)

Quantitative analysis of nestin. $\mathbf{g}$ Quantification of $\beta$-tubulin IIl-positive cells. $\mathbf{h}$ Quantification of GFAP-positive cells. Values are means \pm SD ( $n=3$ /

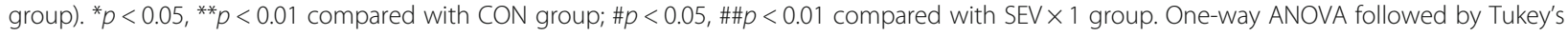
post hoc multiple comparison test was used for data analysis

Values are means $\pm \mathrm{SD}(n=3) .{ }^{*} p<0.05, * p<0.01$, **** $p<0.0001$ compared with CON group; $\# p<0.05$, $\# \# p<0.01, \# \# \# p<0.0001$ compared with SEV $\times 3$ group. One-way ANOVA followed by Tukey's post hoc multiple comparison test was used for data analysis.

ATN1 is the direct target of miR-410-3p

Whether ATN1 is the direct target of miR-410-3p was investigated using TARGETSCAN database [20, 21]. The analysis showed ATN1 was the target site of miR- 410-3p as described in Fig. 7a. The site was further verified using the dual-luciferase reporter assay (Fig. 7b).

\section{Exposure to sevoflurane affects NSC differentiation by} upregulating miR-410-3p

The miR-410-3p level was upregulated in NSCs repeatedly exposed to sevoflurane. The levels of ATN1 (Fig. 8a, f), ß-tubulin III (Fig. 8a, d, g, l), GFAP (Fig. 8a, e, h, m), and nestin (Fig. 8a, i) in NSCs treated with miR-410-3p suppression lentivirus and 

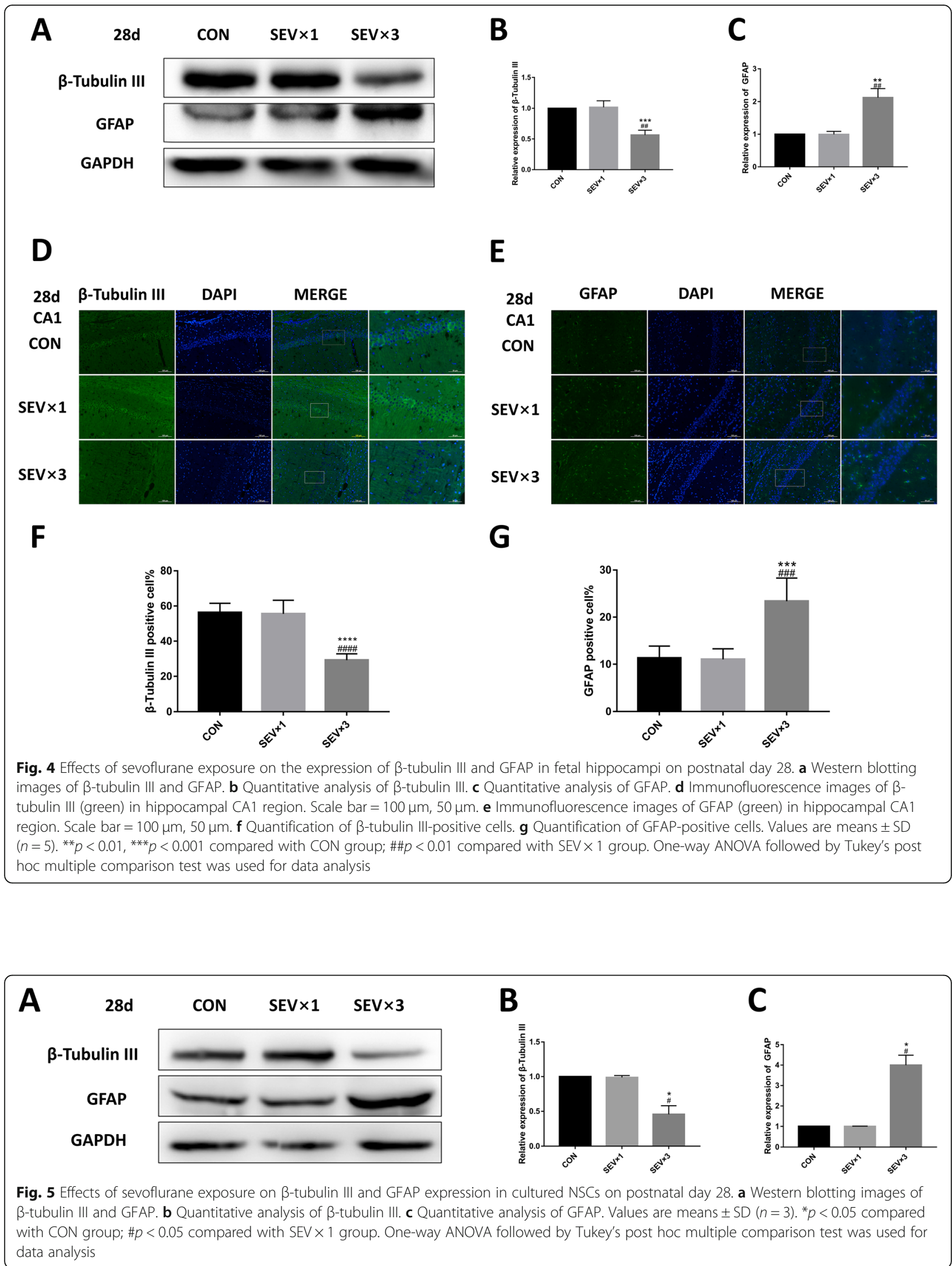


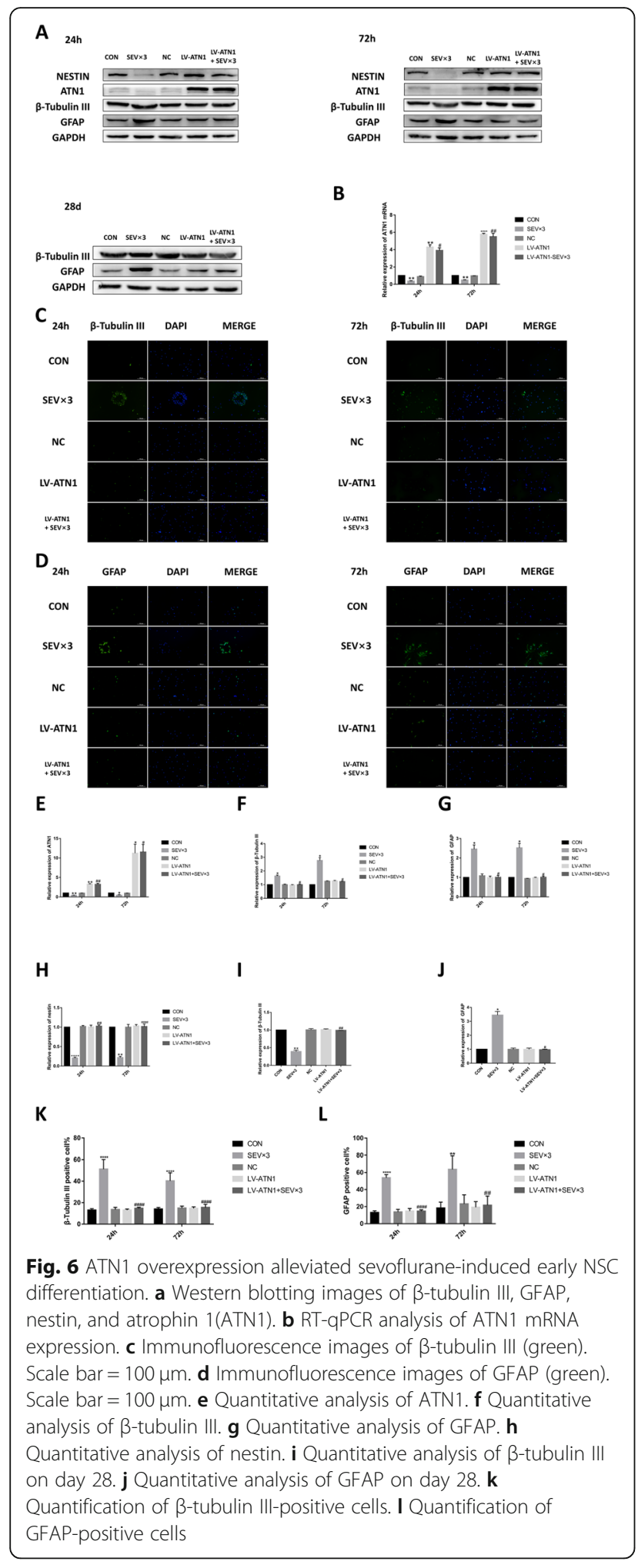

repeatedly exposed to sevoflurane were significantly different than levels in the $\mathrm{SEV} \times 3$ group at $24 \mathrm{~h}$ and $72 \mathrm{~h}$. In the $4.1 \% \mathrm{SEV} \times 3+\mathrm{miR}-410-3 \mathrm{p}$-suppression lentivirus $(\mathrm{LV}-410-\mathrm{SEV} \times 3)$ group, $\beta$-tubulin III expression (Fig. 8a, j) was upregulated and GFAP (Fig. 8a, k) expression was downregulated compared with the SEV $\times 3$ group in cultured NSCs on day 28 . RT-qPCR showed that miR-410-3p was suppressed by lentivirus (Fig. 8b). ATN1 mRNA level in LV-410 + $\mathrm{SEV} \times 3$ group was upregulated compared with the $\mathrm{SEV} \times 3$ group (Fig. $8 \mathrm{c}$ ).

\section{Discussion}

Numerous animal studies have shown that exposure to anesthesia drugs can lead to long-term neurocognitive impairment in developing brains [22, 23]. The FDA has published a warning that neurodevelopment of children's brains may be affected in children under 3 years of age or pregnant women in the third trimester who undergo anesthesia more than once or for more than $3 \mathrm{~h}$ [24]. Mid-trimester is the most common time for non-obstetric surgery; however, the developing brain is in a vulnerable window $[25,26]$ and minor influences such as drugs and environment may cause severe neurocognitive impairment [27-29]. A safe surgery period does not equate to a safe anesthesia period. In the present study, the mechanism of NSC differentiation after exposure to sevoflurane during mid-trimester was investigated and advice provided for further clinical application.

Cell cycle timing dictates cell fate [30]. Premature NSC differentiation may have negative effects on developing brains. In several studies, premature differentiation of NSCs was shown to cause severe brain developmental disorders [31]. Research showed that aneuploidy causes NSCs to exit the cell cycle and differentiate prematurely, eventually resulting in the formation of microcephalic brains [32] In another study, Nap1l1 knockdown caused less BrdU incorporation and greater expression of Tuj $1^{+}$cells (neuronal marker) in embryonic day (E) 13.5 fetal brains, resulting in developmental deficiencies [33]. In maternal diabetes model, the levels of circulating metabolite detoxifying enzyme glyoxalase 1 were decreased, which led to premature neurogenesis and adverse long-term influence on the developing brain of offspring [34].

Although some study results have shown certain anesthesia drugs may affect NSC differentiation, the findings are conflicting and controversial during different periods of brain development. For example, in a previous study, $4.1 \%$ sevoflurane for $6 \mathrm{~h}$ repressed neural differentiation by influencing self-renewal of mouse embryonic stem cells (mESCs) [35]. In another study, 7-day-old rats exposed to ketamine showed significantly reduced number of nestin/BrdU double-stained positive cells and GFAP/BrdU double-stained positive cells and increased number 


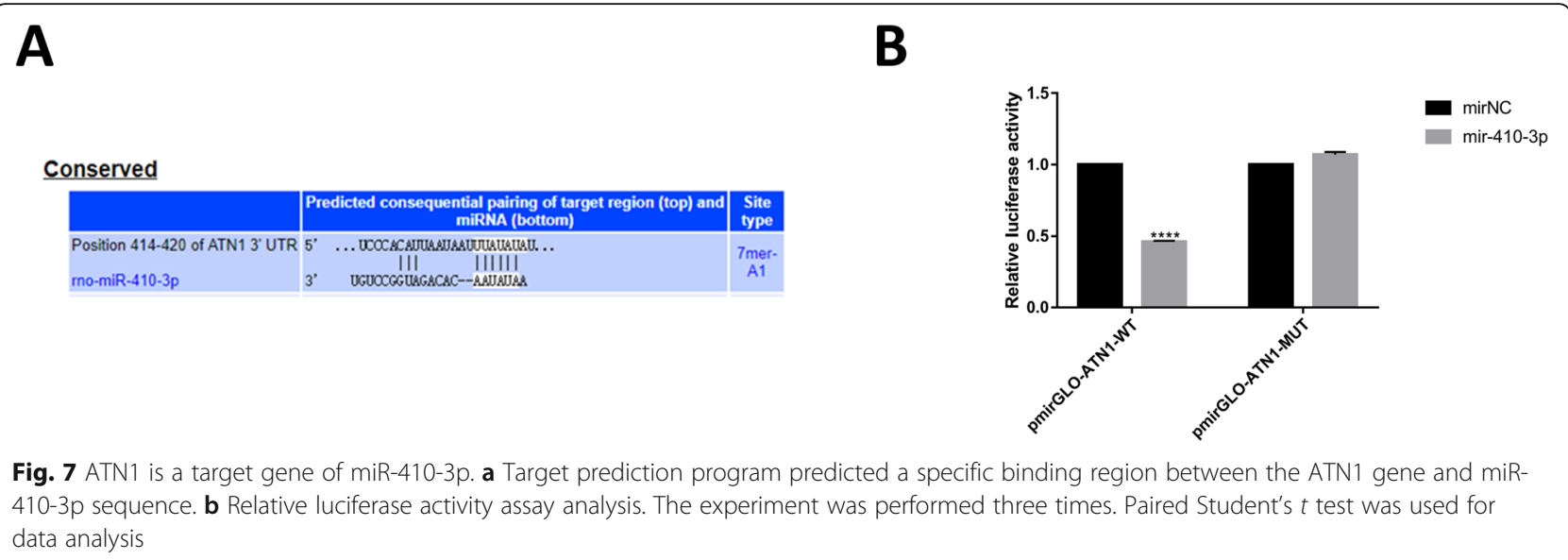

of $\beta$-tubulin III/BrdU double-stained positive cells [36]. Prolonged $2.4 \%$ isoflurane significantly suppressed neuronal fate while promoting glial fate in RcNcell CX human neural progenitor cells [37]. In the present study, 3\% sevoflurane (1.5 minimum alveolar concentration, MAC) was chosen on pregnant rats, a commonly used concentration in clinical nonobstetric surgeries. $4.1 \%$ sevoflurane was commonly used in NSCs to explore the neurotoxicity of SEV in vitro in previous studies $[35,38]$. There are other studies $[39,40]$ using $3 \%$ SEV in vivo and $4.1 \%$ SEV in vitro to investigate the mechanism of SEV. The results showed that during mid-trimester, multiple exposures to sevoflurane can cause premature differentiation of NSCs in developing brains of offspring. A single exposure to $3 \%$ sevoflurane did not significantly influence NSCs after $24 \mathrm{~h}$ and $72 \mathrm{~h}$. After 3 times exposure to $3 \%$ sevoflurane, the expression of NSC marker nestin was downregulated; however, the expression of neuron marker $\beta$-tubulin III and astrocyte marker GFAP was upregulated in the early neurodevelopmental period. The results indicated that multiple exposures to anesthesia can reduce NSCs self-renewal capability and lead NSCs to early differentiation. Over an extended period, $\beta$-tubulin III was suppressed while GFAP remained overexpressed. The reduction of $\beta$-tubulin III indicates the number of neurons was reduced, which supports the same behavioral test results obtained in our previous research showing multiple exposures to sevoflurane can lead to neurocognitive impairment and learning disability in the offspring [19]. Assumedly, sevoflurane causes premature differentiation of NSCs which result in neuronal damage in postnatal off-spring. Consequently, due to the reduction of neurons, gliocytes, such as astrocytes, appear to undergo compensatory proliferation. However, the specific mechanism remains unknown.
Because sevoflurane is one of the most common anesthesia drugs used during pregnancy, the effects on neurodevelopment are crucial. In our previous research, results indicated that sevoflurane can affect proliferation of NSCs and leads to early NSC apoptosis in the offspring [6, 7]. $\beta$-tubulin III stained positive cells and GFAP stained positive cells in proportion to nestin stained positive cells of $\mathrm{SEV} \times 3$ group rates than $\mathrm{CON}$ group which confirms sevoflurane can cause early differentiation of NSCs. Although NSCs have proliferation ability, if they differentiate early to neurons, the proliferation process is disrupted and results in early reduction of neurons. The results from the present study indicate the number of neurons is reduced in the developing brain for an extended period of time after exposure to sevoflurane. Gliocytes tend to proliferate to compensate for the reduction of neurons but cannot reverse the effects on neurocognitive impairment.

The normal ATN1 expression is important for NSC maintenance [12]. Lower ATN1 expression indicates early differentiation of NSCs [12]. After exposure to sevoflurane, ATN1 expression was significantly reduced simultaneously with early NSC differentiation. To detect whether sevoflurane affects NSC differentiation by downregulating ATN1 expression, ATN1 overexpression lentivirus was transfected into primary rat NSCs. The results showed that ATN1 overexpression can reverse the effects of sevoflurane which confirms sevoflurane affected NSC differentiation by regulating ATN1 expression.

MiRNAs play an important role in brain development and miRNAs were affected after exposure to anesthesia drugs in several studies [41, 42]. To further explore the mechanism of NSC differentiation after exposure to sevoflurane, we predicted several possible binding sites using multiple target prediction programs $[20,21]$ and confirmed the sites through 


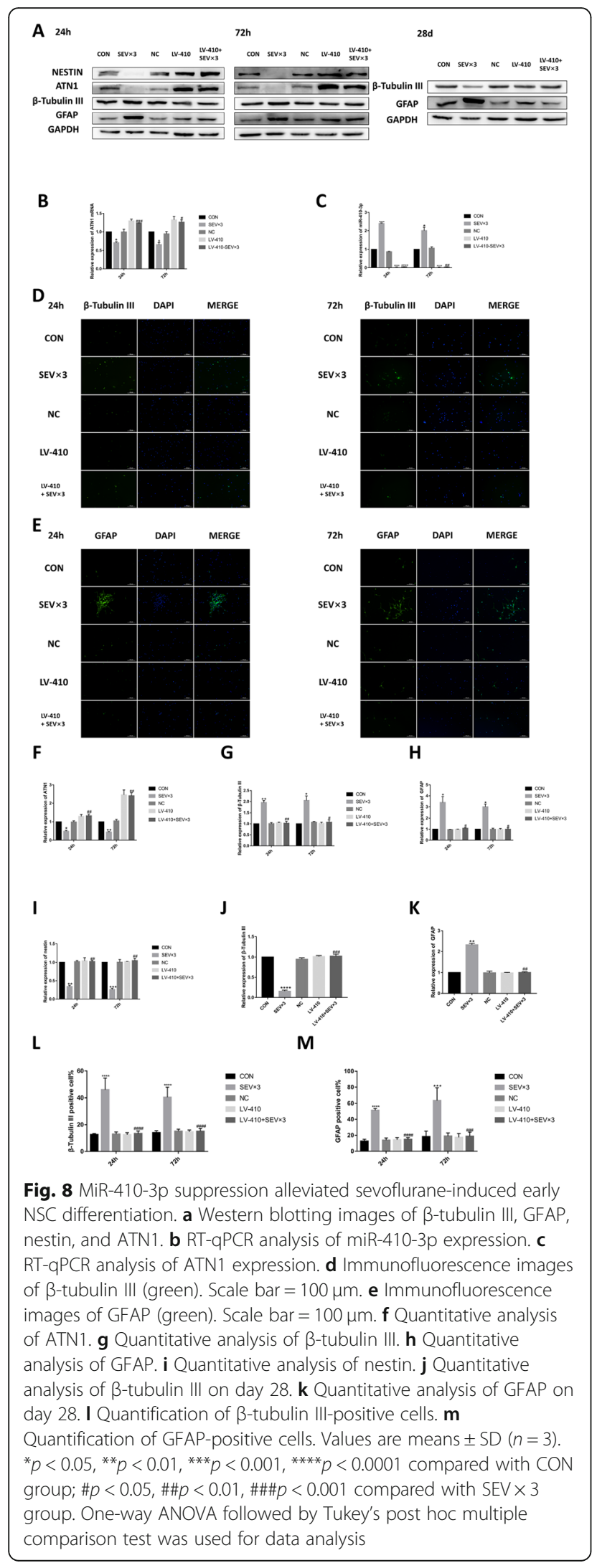

experiments. After exposure to sevoflurane, the miR410-3p expression was significantly upregulated. Dual luciferase reporter assay showed that ATN1 is a direct target of miR-410-3p; miR-410-3p suppression can alleviate the effects of sevoflurane.

In the present study, the focus was on the midtrimester of pregnancy which is the period when NSCs undergo significant proliferation and differentiation. Despite differences among studies, anesthesia drugs were shown in several reports to cause long-term neural impairment $[43,44]$. In the present study, mid-trimester pregnant rats and primary cultured hippocampal NSCs extracted from the same period were used to investigate the influence of sevoflurane on hippocampal NSC differentiation.

The present study had several limitations. First, due to technical limitations, the in vivo mechanism was not investigated. Primary cultured NSCs were used to closely simulate the in vivo environment. Second, the density of cultured NSCs at day 28 was too low to perform immunofluorescence; however, western blot analysis was used to detect the expression of $\beta$-tubulin III and GFAP. Third, only a single inhalational anesthetic was used instead of a drug combination. Combined use of general anesthetics may cause diverse neurotoxicity outcomes, which may be the subject of our future investigations.

\section{Conclusions}

In conclusion, the results from the present study demonstrated that repeated sevoflurane exposure during midtrimester pregnancy caused early differentiation of NSCs by regulating miR-410-3p and ATN1 expression in the offspring. The results may provide several novel strategies for future clinical use.

\section{Supplementary information}

Supplementary information accompanies this paper at https://doi.org/10 1186/s13287-020-01936-9.

Additional file 1: Figure S1. Effects of sevoflurane exposure on the expression of ATN1 in fetal hippocampi. (A) Western blotting images of ATN1 at $24 \mathrm{~h}$. (B) Western blotting images of ATN1 at $72 \mathrm{~h}$.

\section{Abbreviations}

NSC: Neural stem cell; G: Gestational day; GFAP: Glial fibrillary acidic protein; ATN1: Atrophin 1; MiRNAs: MicroRNAs; DRPLA: Dentatorubral-pallidoluysian atrophy; DMEM: Dulbecco's modified Eagle's medium; bFGF: Basic fibroblast growth factor; EGF: Epidermal growth factor; FBS: Fetal bovine serum; SEV: Sevoflurane; E: Embryonic; ANOVA: Analysis of variance; CON: Control group; SOX2: Sex-determining region $Y$ box 2

\section{Acknowledgements}

Not applicable.

\section{Authors' contributions}

$Y Z$ and $P Z$ designed the experiments. $Y Z, Z W$, and $P Z$ contributed to the conception of the work. $Y Z$ performed experiments, collected, and analyzed the data with the help of $Z W, X L, Y W$, and $Y Z$. YZ wrote the manuscript with 
the help of ZW. PZ supervised the study. All authors read and approved the article.

\section{Funding}

This work was supported by the National Natural Science Foundation of China (No.81870838, No.81671311), the Key Research and Development Program of Liaoning Province (No.2018225004), Liaoning Province Distinguished Professor Support Program (No.XLYC1802096), and the Outstanding Scientific Fund of Shengjing Hospital (No.201708).

\section{Availability of data and materials}

All data generated or analyzed during this study are included in this published article and its supplementary information files.

\section{Ethics approval and consent to participate}

All experimental procedures were approved by the Institutional Animal Care and Use Committee of Shengjing Hospital, China Medical University (No.2016PS028K).

\section{Consent for publication}

Not applicable.

\section{Competing interests}

The authors declare that they have no competing interests.

\section{Received: 28 May 2020 Revised: 28 August 2020}

Accepted: 15 September 2020 Published online: 29 September 2020

\section{References}

1. Xu L, Shen J, Yu L, Sun J, Yan M. Autophagy is involved in sevofluraneinduced developmental neurotoxicity in the developing rat brain. Brain Res Bull. 2018;140:226-32. https://doi.org/10.1016/j.brainresbull.2018.05.014.

2. Shenv FY, Song YC, Guo F, Xu ZD, Li Q, Zhang B, Liu ZQ. Cognitive Impairment and Endoplasmic Reticulum Stress Induced by Repeated ShortTerm Sevoflurane Exposure in Early Life of Rats. Fron Psychiatry. 2018;9:3322. https://doi.org/10.3389/fpsyt.2018.00332.

3. Pearn ML, Schilling JM, Jian M, Egawa J, Wu C, Mandyam CD, Head BP. Inhibition of RhoA reduces propofol-mediated growth cone collapse, axonal transport impairment, loss of synaptic connectivity, and behavioural deficits. Br J Anaesth. 2018;120(4):745-60. https://doi.org/10.1016/j.bja.2017.12.033.

4. Meredith RM, Dawitz J, Kramvis I. Sensitive time-windows for susceptibility in neurodevelopmental disorders. Trends Neurosci. 2012;35(6):335-44. https://doi.org/10.1016/j.tins.2012.03.005

5. Silbereis JC, Pochareddy S, Zhu Y, Li M, Sestan N. The Cellular and Molecular Landscapes of the Developing Human Central Nervous System. Neuron. 2016;89(2):248-68. https://doi.org/10.1016/j.neuron.2015.12.008.

6. Wang $Y$, Yin S, Xue H, Yang Y, Zhang N, Zhao P. Mid-gestational sevoflurane exposure inhibits fetal neural stem cell proliferation and impairs postnatal learning and memory function in a dose-dependent manner. Dev Biol. 2018;435(2):185-97. https://doi.org/10.1016/j.ydbio.2018.01.022.

7. Li X, Wu Z, Zhang Y, Xu Y, Han G, Zhao P. Activation of Autophagy Contributes to Sevoflurane-Induced Neurotoxicity in Fetal Rats. Front Mol Neurosci. 2017;10:432. https://doi.org/10.3389/fnmol.2017.00432.

8. Wang Y, Yin SW, Zhang N, Zhao P. High-concentration sevoflurane exposure in mid-gestation induces apoptosis of neural stem cells in rat offspring. Neural Regen Res. 2018;13(9):1575-84. https://doi.org/10.4103/ 1673-5374.237121.

9. Schilling G, Wood JD, Duan K, Slunt HH, Gonzales V, Yamada M, Ross CA. Nuclear accumulation of truncated atrophin-1 fragments in a transgenic mouse model of DRPLA. Neuron. 1999);24(1):275-86.

10. Bidollari E, Rotundo G, Altieri F, Amicucci M, Wiquel D, Ferrari D, Rosati J. Generation of induced pluripotent stem cell line CSSi008-A (4698) from a patient affected by advanced stage of Dentato-Rubral-Pallidoluysian atrophy (DRPLA). Stem Cell Res. 2019;40:101551. https://doi.org/10.1016/j.scr.2019. 101551

11. Napoletan F, Occhi S, Calamita P, Volpi V, Blanc E, Charroux B, Fanto M. Polyglutamine Atrophin provokes neurodegeneration in Drosophila by repressing fat. Embo j. 2011;30(5):945-58. https://doi.org/10.1038/emboj. 2011.1.

12. Zhang F, Xu D, Yuan L, Sun $Y, X u Z$ Z. Epigenetic regulation of Atrophin1 by lysine-specific demethylase 1 is required for cortical progenitor maintenance. Nat Commun. 2014;5:5815. https://doi.org/10.1038/ ncomms6815.

13. Rajman M, Schratt G. MicroRNAs in neural development: from master regulators to fine-tuners. Development. 2017;144(13):2310-22. https://doi. org/10.1242/dev.144337.

14. Shu P, Wu C, Liu W, Ruan X, Liu C, Hou L, Peng X. The spatiotemporal expression pattern of microRNAs in the developing mouse nervous system. J Biol Chem. 2019;294(10):3444-53. https://doi.org/10.1074/jbc.RA118. 004390

15. Shi Z, Zhou H, Lu L, Pan B, Wei Z, Liu J, Feng S. MicroRNA-29a regulates neural stem cell neuronal differentiation by targeting PTEN. J. Cell. Biochem. 2018;119(7):5813-20. https://doi.org/10.1002/jcb.26768.

16. Morgado AL, Rodrigues CMP, Solá S. MicroRNA-145 Regulates Neural Stem Cell Differentiation Through the Sox2-Lin28/let-7 Signaling Pathway. Stem Cells. 2016:34(5):1386-95. https://doi.org/10.1002/stem.2309.

17. Shao CZ, Xia KP. Sevoflurane anesthesia represses neurogenesis of hippocampus neural stem cells via regulating microRNA-183-mediated NR4A2 in newborn rats. J Cell Physiol. 2019;234(4):3864-73. https://doi.org/ 10.1002/jcp.27158.

18. Cao Se, Tian J, Chen S, Zhang X, Zhang Y. Role of miR-34c in ketamineinduced neurotoxicity in neonatal mice hippocampus. Cell Biol Int. 2015: 39(2):164-8. https://doi.org/10.1002/cbin.10349.

19. Wu Z, Li X, Zhang Y, Tong D, Wang L, Zhao P. Effects of Sevoflurane Exposure During Mid-Pregnancy on Learning and Memory in Offspring Rats: Beneficial Effects of Maternal Exercise. Front Cell Neurosci. 2018;12:122. https://doi.org/10.3389/fncel.2018.00122.

20. Agarwal V, Bell GW, Nam JW, Bartel DP. Predicting effective microRNA target sites in mammalian mRNAs. eLife. 2015:4. https://doi.org/10.7554/eLife.05005.

21. Friedman $\mathrm{RC}$, Farh $\mathrm{KKH}_{\text {, }}$ Burge $\mathrm{CB}$, Bartel DP. Most mammalian mRNAs are conserved targets of microRNAs. Genome Res. 2009:19(1). https://doi.org/10. 1101/gr.082701.108

22. Zhong Y, Chen J, Li L, Qin Y, Wei Y, Pan S, Xie Y. PKA-CREB-BDNF signaling pathway mediates propofol-induced long-term learning and memory impairment in hippocampus of rats. Brain Res. 2018;1691:64-74. https://doi. org/10.1016/j.brainres.2018.04.022.

23. Han X, Liu C, Zhang K, Guo M, Shen Z, Liu Y, Li Y. Calpain and JNK pathways participate in isoflurane - induced nucleus translocation of apoptosis-inducing factor in the brain of neonatal rats. Toxicol Lett. 2018; 285:60-73. https://doi.org/10.1016/j.toxlet.2017.12.022.

24. Olutoye OA, Baker BW, Belfort MA, Olutoye OO. Food and Drug Administration warning on anesthesia and brain development: implications for obstetric and fetal surgery. Am J Obstet Gynecol. 2018;218(1). https://doi. org/10.1016/j.ajog.2017.08.107

25. Pletikos M, Sousa AMM, Sedmak G, Meyer KA, Zhu Y, Cheng F, Sestan N. Temporal specification and bilaterality of human neocortical topographic gene expression. Neuron. 2014;81(2):321-32. https://doi.org/10.1016/j. neuron.2013.11.018

26. Vasung L, Abaci Turk E, Ferradal SL, Sutin J, Stout JN, Ahtam B, Grant PE. Exploring early human brain development with structural and physiological neuroimaging. Neuroimage. 2019:187:226-54. https://doi.org/10.1016/j. neuroimage.2018.07.041.

27. Heroux NA, Horgan CJ, Rosen JB, Stanton ME. Cholinergic rescue of neurocognitive insult following third-trimester equivalent alcohol exposure in rats. Neurobiol Learn Mem. 2019:163:107030. https://doi.org/10.1016/j. nlm.2019.107030.

28. Kanlikilicer P, Zhang D, Dragomir A, Akay YM, Akay M. Gene expression profiling of midbrain dopamine neurons upon gestational nicotine exposure. Med Biol Eng Compu. 2017;55(3):467-82. https://doi.org/10.1007/ s11517-016-1531-8.

29. Slotkin TA, Skavicus S, Card J, Levin ED, Seidler FJ. Diverse neurotoxicants target the differentiation of embryonic neural stem cells into neuronal and glial phenotypes. Toxicology. 2016:372:42-51. https://doi.org/10.1016/j.tox.2016.10.015.

30. Dalton S. Linking the Cell Cycle to Cell Fate Decisions. Trends Cell Biol. 2015 25(10):592-600. https://doi.org/10.1016/j.tcb.2015.07.007.

31. Večeřa J, Procházková J, Šumberová V, Pánská V, Paculová H, Lánová MK, Pacherník J. Hypoxia/Hif1a prevents premature neuronal differentiation of neural stem cells through the activation of Hes1. Stem Cell Res. 2020;45: 101770. https://doi.org/10.1016/j.scr.2020.101770.

32. Gogendeau D, Siudeja K, Gambarotto D, Pennetier C, Bardin AJ, Basto R. Aneuploidy causes premature differentiation of neural and intestinal stem cells. Nat Commun. 2015;6:8894. https://doi.org/10.1038/ncomms9894. 
33. Qiao H, Li Y, Feng C, Duo S, Ji F, Jiao J. Nap1l1 Controls Embryonic Neural Progenitor Cell Proliferation and Differentiation in the Developing Brain. Cell Rep. 2018;22(9):2279-93. https://doi.org/10.1016/j.celrep.2018.02.019..

34. Yang G, Cancino GI, Zahr SK, Guskjolen A, Voronova, A, Gallagher D, Miller FD. A Glo1-Methylglyoxal Pathway that Is Perturbed in Maternal Diabetes Regulates Embryonic and Adult Neural Stem Cell Pools in Murine Offspring. Cell Rep. 2016;17(4):1022-36. https://doi.org/10.1016/j.celrep.2016.09.067.

35. Yi X, Cai Y, Zhang N, Wang Q, Li W. Sevoflurane inhibits embryonic stem cell self-renewal and subsequent neural differentiation by modulating the let-7a-Lin28 signaling pathway. Cell Tissue Res. 2016;365(2):319-30. https:// doi.org/10.1007/s00441-016-2394-x.

36. Huang H, Liu L, Li B, Zhao PP, Xu CM, Zhu YZ, Wu YQ. Ketamine Interferes with the Proliferation and Differentiation of Neural Stem Cells in the Subventricular Zone of Neonatal Rats. Cell Physiol Biochem. 2015;35(1):31525. https://doi.org/10.1159/000369698.

37. Zhao X, Yang Z, Liang G, Wu Z, Peng Y, Joseph DJ, Wei H. Dual effects of isoflurane on proliferation, differentiation, and survival in human neuroprogenitor cells. Anesthesiology. 2013;1 18(3):537-49. https://doi.org/ 10.1097/ALN.0b013e3182833fae.

38. Liu S, Fang F, Song R, Gao X, Jiang M, Cang J. Sevoflurane affects neurogenesis through cell cycle arrest via inhibiting wnt/ $\beta$-catenin signaling pathway in mouse neural stem cells. Life Sci. 2018;209:34-42. https://doi. org/10.1016/j.lfs.2018.07.054.

39. Zhang Y, Lu P, Liang F, Liufu N, Dong Y, Zheng JC, Xie Z. Cyclophilin D Contributes to Anesthesia Neurotoxicity in the Developing Brain. Front Cell Dev Biol. 2019:7:396. https://doi.org/10.3389/fcell.2019.00396.

40. Zhang J, Dong Y, Zhou C, Zhang Y, Xie Z. Anesthetic sevoflurane reduces levels of hippocalcin and postsynaptic density protein 95 . Mol Neurobiol. 2015;51(3):853-63. https://doi.org/10.1007/s12035-014-8746-1.

41. Jiang C, Logan S, Yan Y, Inagaki Y, Arzua T, Ma P, Bai X. Signaling network between the dysregulated expression of microRNAs and mRNAs in propofol-induced developmental neurotoxicity in mice. Sci Rep. 2018;8(1): 14172. https://doi.org/10.1038/s41598-018-32474-3.

42. Bahmad HF, Darwish B, Dargham KB, Machmouchi R, Dargham BB, Osman M, Chamaa F. Role of MicroRNAs in Anesthesia-Induced Neurotoxicity in Animal Models and Neuronal Cultures: a Systematic Review. Neurotox Res. 2020;37(3):479-90. https://doi.org/10.1007/s12640-019-00135-6.

43. Liu B, Ou G, Chen Y, Zhang J. Inhibition of protein tyrosine phosphatase 1B protects against sevoflurane-induced neurotoxicity mediated by ER stress in developing brain. Brain Res Bull. 2019;46:28-39. https://doi.org/10.1016/j. brainresbull.2018.12.006.

44. Li GF, Li ZB, Zhuang SJ, Li GC. Inhibition of microRNA-34a protects against propofol anesthesia-induced neurotoxicity and cognitive dysfunction via the MAPK/ERK signaling pathway. Neurosci Lett. 2018;675:152-9. https://doi. org/10.1016/j.neulet.2018.03.052.

\section{Publisher's Note}

Springer Nature remains neutral with regard to jurisdictional claims in published maps and institutional affiliations.

\section{Ready to submit your research? Choose BMC and benefit from}

- fast, convenient online submission

- thorough peer review by experienced researchers in your field

- rapid publication on acceptance

- support for research data, including large and complex data types

- gold Open Access which fosters wider collaboration and increased citations

- maximum visibility for your research: over $100 \mathrm{M}$ website views per year

At $\mathrm{BMC}$, research is always in progress.

Learn more biomedcentral.com/submissions 